\title{
Szenarien Machen Mögliche Zukünfte Erlebbar - Szenen eines Forschungsvorhabens
}

\author{
Maren Ohlhoff, Mehdi Mozuni, Gerhard Glatzel
}

In diesem Beitrag diskutieren wir die Szenariotechnik als partizipatives Instrument zum Umgang mit komplexen, nichtlinearen Systemen. Exemplarisch berichten wir über ein transdisziplinär angelegtes Projekt zur Transformation der Landwirtschaft. Anhand dessen skizzieren wir folgend den Nutzen der Szenariotechnik als auch die Notwendigkeit eines integrativen und inklusiven Vorgehens, um der Komplexität und der damit verbundenen Unsicherheit zur Modellierung zukünftiger Systeme zu begegnen. Das damit eng verbundene Verständnis von nachhaltiger Entwicklung als Zielformulierung verstehen wir als stetigen Aushandlungsprozess und veränderliches Ziel. In diesem Kontext legen wir das Entwerfen multipler Zukünfte als größten Vorteil der Szenariomethode dar. Ebenso argumentieren wir, dass die Szenariotechnik den Ansprüchen an einen nachhaltigkeitsfokussierten Modellierungsansatz gerecht wird und beleuchten sowohl Potenziale als auch Herausforderungen. Der Fokus liegt dabei auf dem Potenzial, mit Hilfe der Szenariotechnik das Wissen unterschiedlicher Akteure aus Wissenschaft und Praxis zu verbinden und zu kommunizieren. Die hier vorgestellte Szeanriotechnik adressiert dabei Systemwissen, Zielwissen und Transformationswissen mit dem Ziel, dieses als partizipativ generierte Erzählung zusammenzuführen.

Keywords: Zukunftsforschung, Szenariotechnik, komplexe Systeme, System Design, Landwirtschaft, nachhaltige Entwicklung

\section{Einleitung}

Weite Felder, Wiesen und Auen, ein Mähdrescher fährt über den Acker, drischt den leuchtenden Weizen in der sengenden Hitze um Spreu und Körner zu trennen. Aus der Ferne ruft ein Rotmilan. Ein Wanderer genießt den weiten Ausblick bis hinüber zu den goldenen Rapsfeldern während inm wohlwärmend der Geruch des frisch gedroschenen Weizens in die Nase steigt.

Eine Geschichte, die sich so oder ähnlich unter Umständen im Ländlichen abspielen mag, aber auch eine gewohnt postulierte Idylle eines Raumes und einer Industrie zeichnet. Je nachdem welche Perspektive man einnimmt, welchem Forschungsparadigma man folgt, welcher Wissensfundus vorhanden ist, ergeben sich andere Fragen oder auch Emotionen auf dieses Bild. Welche Geschichten können und wollen wir über die Zukunft dieser Momentaufnahme erzählen? Wollen Landwirt/innen, Wanderer, wir 
als Gesellschaft und der Rotmilan dieses Bild erhalten, auflösen oder verbessern? Und stimmt dieses Bild überhaupt?

Im Projekt Energy-4-Agri (E4A), welches wir hier als Beispielprojekt vorstellen, geht es weniger um den Status Quo der Landwirtschaft, obschon dieser als Referenz für die Entwicklung von möglichen Zukünften gilt (gefördert durch das Bundesministerium für Wirtschaft und Energie; Projektträger Jülich, Forschungszentrum Jülich; Laufzeit: 01.04.2020-31.03.2023; Projektwebsite: https://www.tu-braunschweig.de/energy-4agri). Die gemeinsame Forschungsfrage im Projekt lautet: Wie könnte eine zukünftige Landwirtschaft mit elektrischen Antrieben aussehen und welche Potenziale und weiteren Wirkungen entstehen daraus? Dazu arbeiten zwei Hochschulen in einem transdisziplinären Forschungsvorhaben aus den Bereichen Landtechnik, Energietechnik, Geoökologie, Psychologie und Design eng zusammen (Beteiligte Institute der TUBraunschweig: Institut für mobile Maschinen und Nutzfahrzeuge (IMN); Geoökologie Institut für Geoökologie (IGÖ); Abteilung für Arbeits-, Organisations- und Sozialpsychologie (AOS) am Institut für Psychologie; elenia Institut für Hochspannungstechnik und Energiesysteme (elenia); Beteiligtes Institut der HBK-Braunschweig: Institut für Designforschung (IDF)). Die Forschungsfrage des Projektes wirft ein Licht auf zu lösende Probleme, die i.d.R. komplex und nichtlinear sind. Weder einfache noch vereinfachende Lösungen sind zielführend. Linear extrapolierende Lösungspfade helfen nur in Ausnahmefällen, Erfahrungswissen hilft nur bedingt, da es sehr häufig in einem veralteten System erworben wurde. Doch das Nachdenken über Zukunft wird im Wesentlichen geprägt durch Erfahrungen (Pillkahn: 2013, 52). Ein Lösungssystem muss mindestens die gleiche Komplexität aufweisen wie das Problemsystem. Methoden sollten daher perspektivische Erfahrungen und Fachwissen bündeln und diese letztlich auch für Nichtexperten aufbereiten können. In diesem Beitrag argumentieren wir, dass die Szenariotechnik als Instrument genutzt werden kann, um die genannten Anforderungen zu erfüllen. Ziel ist das Design und die Modellierung multipler zukünftiger Systemzustände. Das Forschungsparadigma beschreibt Jonas (Jonas: 2007, 30) mit Forschung durch Design mittels Szenarien. Die Gestaltung dient als Lieferant von Transformationswissen und einer teambildenden und identitätsstiftenden gemeinsamen Erzählung einer gelingenden Transformation. Mittels Designmethoden erstellten Szenarien stellen das die sehr diversen Disziplinen verbindende und inhaltliche Forschungsziele definierende Element dar. 


\section{Komplexität handhaben: Denken in Systemen - Denken in Szenarien}

Es lässt sich recht einfach postulieren und theoriegeleitet belegen, dass und warum ein Denken in Systemen zur Lösung von komplexen Problemen sinnvoll ist. Allerdings zeigt sich trotz aller Hinweise eine mangelnde Erfolgsbilanz von Nachhaltigkeit oder nachhaltiger Entwicklung in der praktischen Umsetzung (Milbert 2013, 37 f.). Dies lässt sich laut Milbert (ebd.) auf die Komplexität der betrachteten Systeme zurückführen oder vielmehr auf eine Kapitulation vor der Komplexität. Unter anderem aufgrund der Tatsache, dass Nachhaltigkeit als Ziel nicht allein wissenschaftlich erreichbar, sondern das Ergebnis gesellschaftlicher Aushandlungsprozesse ist. Für einen praxisorientierten komplexitätsbasierten Modellierungsansatz hinsichtlich einer nachhaltigen Transformation schlagen Peter \& Swilling $(2014,1612)$ explizit vor, Szenarien zu generieren, um (u.a.) Wahrscheinlichkeiten mit einzubeziehen und der Nichtlinearität Rechnung zu tragen. Dies betrifft auch den Faktor Unsicherheit, denn die Kenntnisse über einzelne Systeme (ob nun ökologisch, ökonomisch oder sozial) und ihr Verhalten sind unvollständig und unbestimmt. Das Verhalten eines Systems kann daher nur probabilistisch ausgedrückt werden (Clayton \& Radcliffe: 1996, 36). Ein zukunftsgerichtetes Systemdenken sollte darüber hinaus fragen, wie ein gut angepasster Systemzustand definiert werden kann und für wen dies ein gut angepasster Zustand wäre (Skrimizea, Haniotou \& Parra: 2019, 135). Szenarien verkörpern damit die Grundeinstellung, "dass Zukunft zwar nicht vorhersehbar, wohl aber zu wesentlichen Teilen gestaltbar ist" (Gaßner: 2013, 412). Dazu sei angemerkt, dass auch Prognosen keine Vorhersagen bilden können. Prognosen werfen i. d. R. einen Blick auf die vergangene Entwicklung und schreiben diese linear fort. Szenarien beziehen zwar Prognosen und Trends mit ein formulieren aber auch direkt erwünschte und unerwünschte Zustände (siehe Abb.1). Dies hebt die Notwendigkeit eines transdisziplinären Vorgehens hervor. Neben der Einbindung von Wahrscheinlichkeit sollten Modellierungsansätze daher auch integrativ, inklusiv sowie adaptiv sein (Peter \& Swilling: 2014, 1597).

Was aber hat die Szenariotechnik über den partizipativen Aspekt hinaus zu bieten, wenn es um Komplexität geht? Je nach angewendeter Szenariotechnik lässt sich der Komplexitätsgrad unterschiedlich erfassen: Kosow, Gaßner, Erdmann \& Lubner (2008, 78) stellen fest: „Simple trendbasierte Szenariotechniken vernachlässigen häufig Wechselwirkungen." Auch Pillkahn $(2013,52)$ erkennt die Tendenz, dass der Großteil der Trend- und Zukunftsforscher Komplexitäten ausblendet. Veränderungen würden dabei bevorzugt linear mit Hilfe von Trends dargestellt, da "sie so einfach, so erklärend 
und komplexitätsreduzierend wirken [...]". Ein Trend der heute als sicher gilt, kann jedoch morgen schon wieder obsolet sein. Ebenso ist nachhaltige Entwicklung als Zielvorstellung veränderlich und mit Unsicherheiten verknüpft (Milbert: 2013, 37 f.). Entsprechend ist das Einzige, was wir sicher wissen: Das System Landwirtschaft wird sich bis 2050 verändert haben. Gegenüber Trendszenarien erlauben systematisch-formalisierte Verfahren (z. B. die Konsistenzanalyse) eine detaillierte Betrachtung einzelner Entwicklungen. Allerdings ist dies auch mit einer Einschränkung auf eine bestimmte Anzahl von Schlüsselfaktoren (10 bis maximal 25) verbunden (Kosow, Gaßner, Erdmann \& Lubner: 2008, 78). „Kreativ-narrative Verfahren haben demgegenüber den Vorteil, weniger explizit Schlüsselfaktoren auswählen zu müssen, und können deshalb ggf. mehr Aspekte, Nuancen und Faktoren beachten, tun dies aber häufig weniger detailliert oder weniger systematisch“ (ebd.). Aus diesen Gründen setzen wir im Projekt E4A sowohl stark formalisierte als auch intuitiv-kreative Verfahren ein.

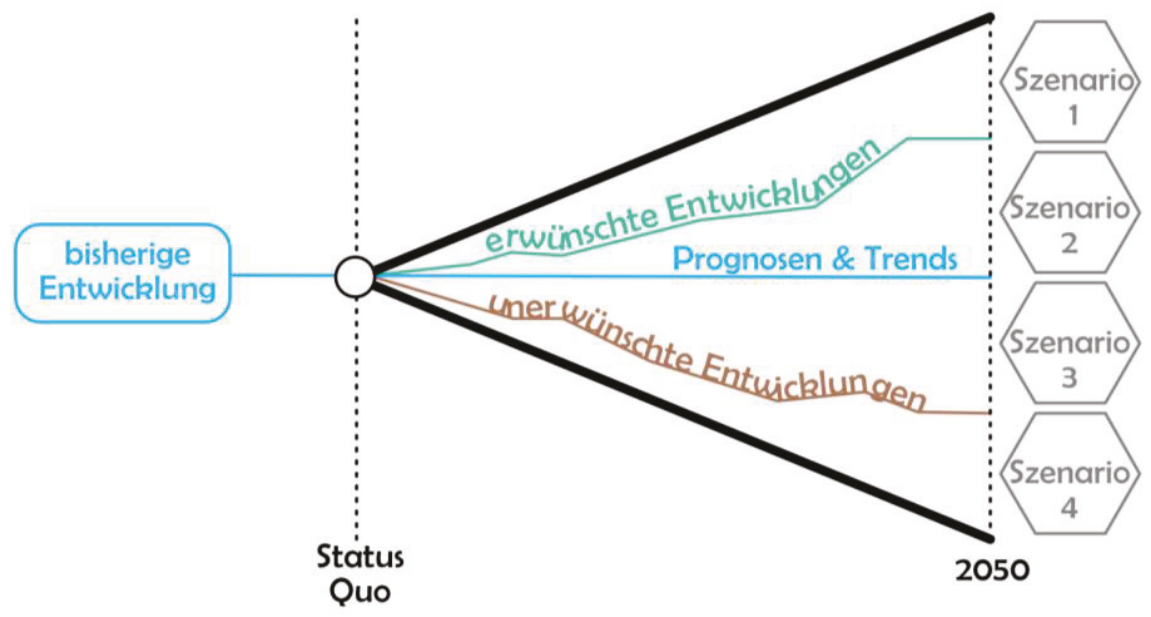

Abbildung 1: Szenarienentwicklung

\section{Wissen verbinden}

Im Projekt E4A ist das Ziel der Teilprojektarbeiten in den beteiligten Instituten, zu den Bereichen Agrartechnik, Energietechnik und Landschaftsökologie u.a technische Simulationen zum Energieverbrauch, zur Energieerzeugung und zu den ökologischen Auswirkungen (z. B. Kleintechnik vs. Großtechnik) durchzuführen. Die Psychologie unter- 
stützt das Vorhaben über die Einbindung von Stakeholdern hinsichtlich Akzeptanzforschung und Technikfolgenabschätzungen. In multi- und transdisziplinär angelegten Projekten dient die Szenariotechnik als Instrument diverse Disziplinen und Perspektiven auf vielfältige Weise zu verbinden (dazu vertieft Glatzel \& Wiehle: 2019). Denn „Zukünfte entwickeln sich im Allgemeinen nicht entlang einzelner Disziplinen und sind deshalb auch nicht von einzelnen Disziplinen in ihrer Komplexität und vernetzten Funktionalität zu erfassen“ (Kreibich: 2013, 357).

Im konkreten Fall eines Transformationsprojektes wie E4A wird ein Projektteam zusammengestellt, das den disziplinären Kern der Aufgabe und die wesentlichen mitbetroffenen Disziplinen abdeckt. Das heißt, bereits in der Projektdefinitionsphase finden wesentliche projekterfolgsbestimmende Entscheidungen statt. Die Gesamtaufgabe ist in Arbeitspakete gegliedert, die inhaltlich und organisatorisch verknüpft sind, aber ein individuelles Arbeiten unterstützen. Die Inklusion und Integration der Disziplinen mittels Szenarien erfolgt sowohl inhaltlich als auch methodisch. Darüber hinaus findet eine Einbindung von weiteren ExpertInnen und Stakeholdern statt. Das Vorhaben ist daher eng mit einzelnen Landwirtschaftsbetrieben, institutionellen Interessengruppen wie der niedersächsischen Landwirtschaftskammer und weiteren Forschungseinrichtungen verknüpft. Regelmäßige Meilensteintermine und ein gemeinsamer Beirat unterstützen die Definition gemeinsamer Ziele. Dadurch werden unterschiedliches Fachwissen und sowohl qualitative als auch quantitative Forschungsergebnisse sowie Pluralitätswissen durch „Expertelnnen“ aber auch „Laien“ (Carceller-Maicas: 39, 307) in die Szenariengenerierung einbezogen. Dieses Pluralitätswissen lässt sich im Kontext transdisziplinärer Forschung auf drei Fragetypen aufteilen: „Ein erster Fragetyp betrifft die Ursachen des Problems und die weitere Entwicklung (System Knowledge - Analyse). Ein weiterer betrifft die Werte und Normen, um Ziele für den Problemlösungsprozess zu gestalten (Target Knowledge - Projektion). Ein dritter bezieht sich auf die Transformation und Verbesserung der Problemsituation (Transformation Knowledge - Synthese)" (Jonas \& Rammler: 2013, 336).

\section{System Knowledge}

Um ein Systemverständnis zu erhalten, haben wir im Projekt E4A mit allen ProjektpartnerInnen eine Sensitivitätsanalyse (nach Vester: 2002, 185 ff.) durchgeführt. Gesucht werden hier diejenigen Faktoren, welche einen großen Einfluss innerhalb des Systems Landwirtschaft nehmen. Das Ergebnis stellt eine 4-Felder Matrix möglicher Zukünfte dar, die sich inhaltlich durch die Faktoren „Wirtschaftlichkeit des Elektrifizierungskonzeptes" und "Politische Maßnahmen“ unterscheidet (Stand März 2021). Eine solche Matrix dient als erster Ausgangspunkt für ein kreativ-narratives Vorgehen bei dem vier 
unterschiedliche Welten mit unterschiedlichen Anforderungen an eine 100\% Elektrifizierung der Landwirtschaft unter Einbindung eines Gegenszenarios skizziert werden. Bei der Ausarbeitung gilt es, Trends und etwaige Gegentrends in die Narrationen mit einzubinden. Parallel erfolgt eine systematisch-formalisierte morphologische Analyse: In einem iterativen Prozess werden mittels Experteninterviews und Workshops disziplinär Schlüsselfaktoren definiert. Ziel ist es, nachfolgend eine Konsistenzanalyse durchzuführen. Das heißt, die BewerterInnen beurteilen, welche Faktoren sich in einem Zukunftsbild gegenseitig ein- oder ausschließen: Wenn z.B. weiterhin große Maschinen die Feldarbeit übernehmen, ist gleichzeitig keine kleinparzellierte Landwirtschaft möglich. Das Anfangs gezeichnete Bild der weiten Felder bliebe bestehen. Es entsteht ein morphologischer Kasten (siehe Abb. 2) konsistenter Zukünfte, der weitere soziale, politische und ökonomische Faktoren umschließt und verbindet. Hinzu kommt die Einbindung des Outputs vom kreativ-narrativen Verfahren als Schlüsselfaktoren. Dies verfolgt die Ziele der gegenseitigen Evaluierung der Outputs beider Verfahren und der Verbesserung beim Erfassen und Beschreiben eines komplexen Systems. Die Vorbereitung, d.h. Festlegung von Schlüsselfaktoren, erfolgt in mehreren iterativen Schritten.

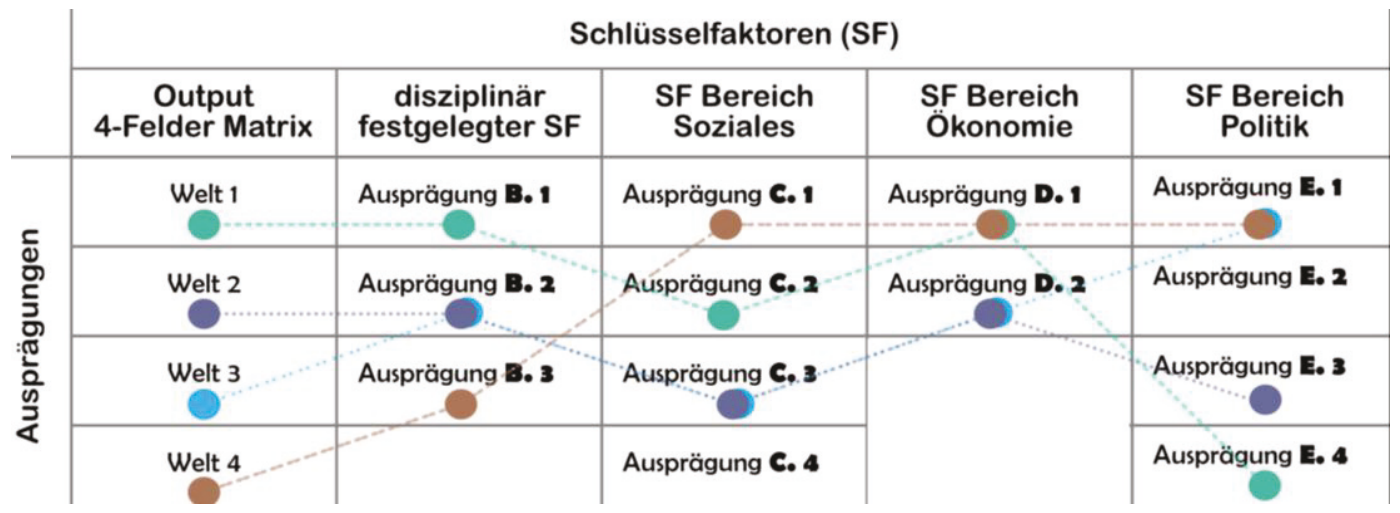

Abbildung 2: Schematische Darstellung eines morphologischen Kastens (exemplarisch)

Systemverständis bedeutet dabei auch Problemverständnis. Eventuelle Probleme entstehen dabei aus dem komplexen Verhalten der einzelnen Subsysteme (Tippett: 2010, 256). Solche Probleme sind im Wesentlichen ein nicht adaptives oder anderweitig unerwünschtes Systemergebnis (Clayton \& Radcliffe: 1996, 191; Steele: 1991, 454). Diese Systemergebnisse sind pfadabhängig. „Ereignisse und Entwicklungen in der Vergangenheit haben Auswirkungen auf den aktuellen und zukünftigen Verlauf der Trajektorie, ohne diesen jedoch zu determinieren und vorhersehbar zu machen" (Weig: 2016, 
266). Für die Lösungsfindung bedarf dies der Erfassung des Systemcharakters als Ganzes (Vester: 2002, 17f.). Als problematisch diskutiertes Thema zum System Landwirtschaft gilt beispielsweise der Biodiversitätsverlust. Dieses Problem lässt sich auf unterschiedliche Ursachen zurückführen (Niggli et al.: 2020,4) und dient folgend als Beispiel für ein zusammengesetztes komplexes Problem. So zeigt sich, dass die Ursachen (z.B. Vereinfachung der Fruchtfolgen, Vergrößerung der Bewirtschaftungsparzellen (ebd., 5)) selbst unerwünschte, emergente Phänomene sind: Beispielsweise ist Weizen heute die wichtigste Getreidesorte in Deutschland, welche den größten Anteil der Anbauflächen einnimmt (BLE: 2020,13). Ein Resultat, das aus der Systemtrajektorie erfolgt, aber gleichzeitig auch durch aktuelle Entwicklungen und Annahmen zur Zukunft beeinflusst wird, wie z.B.:

- Vergangenheit: Domestikation in den Kinderschuhen der Landwirtschaft

- Gegenwart: Agrarmarktpreise, politische Maßnahmen

— Zukunft: Prognosen zur Preisentwicklung

Es ist anzuerkennen, dass komplexe Systeme und deren Auswirkungen sich auch ohne Eingriff und Absicht oder Zielsetzung entwickeln, so war es sicherlich nie Ziel, dass Weizen die wichtigste Getreidesorte wird oder dass immer größere Bewirtschaftungszellen entstehen. Es gilt also auch mögliche Nebenfolgen bei der Systemgestaltung zu betrachten. Selbst das Ziel Erhalt der Biodiversität, als (gesellschaftlich anerkanntes) Nachhaltigkeitskriterium, kann als Reflex (dazu auch Kehren \& Winkler: 2019, 377) auf den beobachtbaren Verlust verstanden werden.

\section{Target Knowledge}

Target Knowledge lässt sich auf zwei Ebenen betrachten. (1) Festlegung von Forschungszielen: Durch die disziplinäre Festlegung von Einfluss- und Schlüsselfaktoren, wie zuvor beschrieben werden gemeinsam System und Systemgrenze in einem iterativen Prozess definiert. Dies bildet den Kern der gemeinsamen multidisziplinären Zusammenarbeit. (2) Festlegung oder vielmehr Diskussion von inhaltlich-normativen-Zielen: Dies impliziert, wie skizziert, ein normatives Verständnis von Nachhaltigkeit. Es geht um die Frage, wie ein gut angepasster Systemzustand, d. h. eine nachhaltige Landwirtschaft, in der Zukunft aussehen könnte. Neben der Konsistenzfrage (welche Zukunft ist möglich und plausibel) gilt es erwünschte und unerwünschte Zukünfte zu erörtern (welche Zukunft wäre für wen wünschenswert?). Dies erfordert die Einbindung von unterschiedlichen Stakeholdern sowie weiteren Experten und Expertinnen. Die Frage nach der Wünschbarkeit bezieht sich gleichermaßen auf Ziel und Prozess. Wie wichtig diese Unterscheidung und Betrachtung ist, zeigt beispielsweise eine Studie 
zur Energiewende: Zwar werden politische Zielsetzungen zur Energiewende mehrheitlich unterstützt, jedoch erfährt der Weg zur Zielerreichung starke Kritik (Wolf: 2020, 2). Ablehnung kann dabei sehr subjektiv sein. Je nach Perspektive können bestimmte Entwicklungen positiv oder negativ gedeutet werden (Kosow, Gaßner, Erdmann \& Luber: $2008,6)$. Wir argumentieren, dass durch das eröffnen alternativer Zukünfte und Strategien auch das Spektrum an Diskussionsgrundlagen erweitert wird, was dabei helfen kann, Unentscheidbarkeiten z.B. zwischen den Zielen ökologisch vs. anthropozentrisch (dazu vertieft Peter \& Swilling: 2014, 1600 f.) aufzudecken und ggf. aufzulösen.

\section{Transformation Knowledge}

Die Szenariengenerierung bezieht sich wie zuvor erörtert nicht nur auf einen bestimmten Zeitpunkt in der Zukunft, sondern auch auf Entwicklungen der Vergangenheit und Gegenwart. Bei der Szenarienarbeit zeigt sich, dass das Nachdenken über Zukunft mit Herausforderungen behaftet ist: „Unsere Vorstellungskraft, die im Wesentlichen auf unseren Erfahrungen beruht, begrenzt uns im Nachdenken über die Zukunft: Nur weil wir uns nicht vorstellen können, dass sich etwas verändert, heißt das [jedoch] noch nicht, dass sich nichts ändert (Pillkahn: 2013, 52). Welches Szenario, welche Entwicklung tatsächlich realistisch(er) oder wahrscheinlicher für das Jahr 2050 wäre, lässt sich somit nicht vorhersagen. Die bisherige Agrarpolitik lässt den Gedanken aufkommen, dass eine grundlegende Transformation eher unwahrscheinlich ist. Für die Zielsetzung einer ökologische(re)n EU-Agrarpolitik wäre nach Isermeyer $(2014,23)$ ein möglicher (aber seines Erachtens unwahrscheinlicher) Weg, den Diskurs weniger auf die Geldverteilung zu legen, sondern auf die Zielsetzungen wie z.B. Erhalt der Biodiversität. Dies würde auch eine entsprechende Operationalisierung und Strategieplanung zur Erreichung des Zieles erfordern. Allerdings resümiert Isermeyer (ebd.), dass seitens der Politik weder ein klares Ziel noch ein zielgerichteter Maßnahmenkatalog bestünde. Demgegenüber würden "eine ganze Reihe von Maßnahmen und Instanzen [etabliert] in der vagen Hoffnung, dass die Probleme damit irgendwie gelöst werden." Die hier formulierte Problematik ist gleich mehrfach relevant, zum einen legt das Beispiel nahe, dass Komplexität hier bei der politischen Lösungsfindung vernachlässigt wurde. Zum anderen werden dadurch die Praxisrelevanz von (normativen) Zielsetzungen und letztlich auch Visionen sowie die Ausformulierung eines Transformationsfades bestätigt, um dieses Ziel zu erreichen.

Dabei spielt die Frage nach der Transformationsgeschwindigkeit bei der Gestaltung und Bewertung eine besondere Rolle: So zeigt sich beispielsweise anhand der Reaktionen auf Maßnahmen bei schnell eintretenden Ereignissen (Beispiel Corona-Pandemie) sehr deutlich, dass diskontinuierliche und disruptive Veränderungen einen hohen 
Widerstand auslösen. Ebenso würde eine plötzliche Veränderung der Agrarpolitik (Abschaffung von Direktzahlungen) vermutlich viele Widerstände auslösen, im Gegensatz zu einem kontinuierlichen Wandel, bei dem Ziel und Weg auch frühzeitig kommuniziert werden würden. Geschichten und Geschichtenerzählen können dabei als Hilfsmittel und Inspiration dienen und neue Vorstellungen von Alternativen entwickeln um Transformation zu gestalten (Sandercock: 2010, 25).

\section{Wissen erzählen}

Auch wenn transdisziplinäre Forschung immer darauf setzt, disziplinäres Wissen zu verbinden (inklusiv) und Probleme auf holistische Weise anzugehen (integrativ) (Carceller-Maicas: 2015, 307), müssen wir jedoch anerkennen, dass wir nie absolutes Wissen erlangen werden (Clayton \& Radcliffe: 1996, 212; Skrimizea, Haniotou \& Parra: 2019, 129). Bei der Ausarbeitung und Beschreibung möglicher Zukünfte handelt es sich daher letztlich auch um reduktionistische Modelle. Modelle (d.h. auch andere Methoden und Techniken) sind per Definition reduktionistisch. Informationsverluste müssen akzeptiert werden, um Einfachheit, Klarheit und Verständnis über den Forschungsgegenstand zu erreichen (Clayton \& Radcliffe: 1996, 190; Ratter \& Treiling: 2008, 26) "We can not deal with reality in all its complexity" (Cilliers: 2008, 50 f.). In dieser Hinsicht hat die Szenariotechnik den Vorteil, dass grundsätzlich mehrere alternative Zukünfte modelliert werden (Kosow, Gaßner, Erdmann \& Luber: 2008, 6; Kosow \& León: 2015, 219) und somit auch ein multiples Strategiespektrum eröffnet wird (Braun, Glauner \& Zweck: 2005, 34), das zur weiteren Diskussion einlädt. Hinzu kommt der iterative und damit auch adaptive Charakter: Durch die stetige Einbindung von Experten und Stakeholdern werden das Forschungsfeld stetig evaluiert und inhaltliche Ziele kontinuierlich ausgehandelt. Zudem können je nach Forschungsfeld unterschiedliche Methoden zur Stakeholdereinbindung unter dem Dach der Szenariotechnik angewendet werden.

Das Potenzial multi- bzw. transdisziplinär erarbeiteter Szenarien liegt darin, Informationen und gemeinsames Wissen besser zu vermitteln und gesellschaftliche Diskurse zu erleichtern (Greeuw: 2000, 9 f.). Somit können Szenarien auch als bessere Alternative zum klassischen Forschungsbericht gesehen werden, um Informationen zu verbreiten (Glatzel und Wiehle: 2019, 336). Durch die Einbindung von diversen Akteuren, Wissen und Perspektiven bewirken die Szenarien darüber hinaus im Optimalfall einen Interessenausgleich zwischen möglichen Interessensgruppen sowie Akzeptanz und Verständnis für die erzeugten Lösungswege und Konzepte. Szenarien im E4A-Projekt legen den Blick auf zwei Erzählungen: Erstens werden mehrere mögliche Systemzustände skiz- 
ziert (Target Knowledge), zweitens werden Alternativen durchgespielt, was vom heutigen Zeitpunkt aus passieren muss (oder nicht passieren sollte), um einen bestimmten Systemzustand zu erreichen (Transformation Knowledge). Beide Geschichtsstränge bauen wiederrum auf dem Systemwissen auf. Dem Umstand der Komplexität wird partizipativ, kreativ und systematisch begegnet, hier im Besonderen durch die Kombination mehrerer Szenarioverfahren. „Szenarien machen mögliche Zukünfte erlebbar“ bedeutet hier auch, Komplexität erlebbar machen. Geschichten haben ein Potenzial, Verständnis für ein komplexes Forschungsfeld zu schaffen, dabei ist es ist jedoch wichtig, die Wahrheit hinter den Geschichten stetig zu hinterfragen (Sandercock 2010, 19 f.). Die Frage, was für wen ein guter Zustand wäre, gewinnt in diesem Sinne weiter an Bedeutung.

\section{Fazit und Ausblick}

Wie skizziert, kann die hier vorgestellte Szenariotechnik dem Methodenanspruch im Kontext eines Nachhaltigkeitsgedankens (probabilistisch, integrativ, inklusiv, adaptiv) gerecht werden. Dabei wird das Vorgehen zwar als reduktionistisch anerkannt, der große Vorteil liegt jedoch in der Schaffung multipler Zukünfte und der Untersuchung unterschiedlicher Transformationspfade. Somit entstehen nicht nur eine Geschichte, eine Vision, ein Bild, sondern gleich mehrere Zukunftsoptionen. Diese laden dazu ein, nachhaltige Landwirtschaft zu diskutieren und zu gestalten und decken gleichzeitig auch nicht nachhaltige Wege auf.

Was es gilt herauszufinden ist, welche Geschichten über eine zukünftige Landwirtschaft und gelingende Transformation wir erzählen wollen. Im Kontext des Nachhaltigkeitsverständnisses gilt es dies im Projekt E4A noch auszuhandeln

\section{Literaturverzeichnis}

BLE - Bundesanstalt für Landwirtschaft und Ernährung (2020): Bericht zur Markt-und Versorgungslage. Getreide 2020. https://www.ble.de/SharedDocs/Downloads/DE/BZL/Daten-Berichte/Getreide_Getreideerzeugnisse/2020BerichtGetreide.pdf?_blob=publicationFile\&v=2, abgerufen am 08.03.2021.

Braun, A., Glauner, C. \& Zweck, A. (2005): Einführung in die Praxis der "Regionalen Vorausschau“. Hintergründe und Methoden. Düsseldorf (ZTC Working Paper Nr. 2). https://www.vditz.de/fileadmin/media/publications/pdf/Praxis_der_Regionalen_Vorausschau.pdf, abgerufen am 01.03.2021.

Carceller-Maicas, N. (2015): Sharing Wisdom(s) to Enrich Knowledge. In: Collegium Antropologicum, 39 (2), 307-315.

Cilliers, P. (2008): Complexity Theory as a general framework for sustainable science. In: Burns, M. \& Weaver, A. (Hrsg.): Exploring sustainability science. A southern African perspective, 39-58, Stellenbosch: African Sun Media. 
Clayton, A. M. H. \& Raddliffe, N. J. (1996): Sustainability. A systems approach. Abingdon, Oxon: Earthscan.

Gaßner, R. (2013): Zukunft als gesellschaftliche Gestaltungsaufgabe. Die Arbeit mit normativen Szenarios. In: Zweck, A. \& Popp, R. (Hrsg.): Zukunftsforschung im Praxistest, 409-416, Wiesbaden: Springer.

Glatzel, G. \& Wiehle, M. (2019): Szenarien verbinden. In: Stelzer, R. H. \& Krzywinski, J. (Hrsg.): Entwerfen Entwickeln Erleben in Produktentwicklung und Design, 327-338, Dresden: TUDpress.

Greeuw, S. C. H. (2000): Cloudy crystal balls. An assessment of recent European and global scenario studies and models. Copenhagen: European Environment Agency.

Isermeyer, F. (2014): Künftige Anforderungen an die Landwirtschaft - Schlussfolgerungen für die Agrarpolitik. Thünen Working Paper 30. Braunschweig: Johann Heinrich von Thünen-Institut, Bundesforschungsinstitut für Ländliche Räume, Wald und Fischerei. https://www.thuenen.de/media/publikationen/thuenen-workingpaper/ThuenenWorkingPaper_30.pdf, abgerufen am 04.03.2021.

Jonas, W. \& Münch, J. (2007): Forschung durch Design als integratives Prozessmodell - eine Skizze. In: Freunde und Förderer des Technischen Designs an der TU Dresden e. V. (Hrsg.): Technisches Design in Forschung, Lehre und Praxis: Symposium Technisches Design Dresden 2007, 19-34, München: Dr. Hut München.

Jonas, W. \& Rammler, S. (2013): „Das Rad neu erfinden“. Forschung zu zukunftsfähiger Mobilität am Institut für Transportation Design Braunschweig. In: Zweck, A. \& Popp, R. (Hrsg.): Zukunftsforschung im Praxistest, 321-352, Wiesbaden: Springer.

Kehren, Y. \& Winkler, C. (2019): Nachhaltigkeit als Bildungsprozess und Bildungsauftrag. In: Leal, W. (Hrsg.): Aktuelle Ansätze zur Umsetzung der UN Nachhaltigkeitsziele, 373-392, Berlin: Springer Berlin; Springer Spektrum.

Kosow, H., Gaßner, R., Erdmann, L. \& Luber, B.J. (2008): Methoden der Zukunfts- und Szenarioanalyse. Überblick, Bewertung und Auswahlkriterien. Berlin: IZT.

Kosow, H. \& León, C. (2015): Die Szenariotechnik als Methode der Experten- und Stakeholdereinbindung. In: Niederberger, M. \& Wassermann, S. (Hrsg.): Methoden der Experten- und Stakeholdereinbindung in der sozialwissenschaftlichen Forschung, 217-242, Wiesbaden: Springer VS.

Kreibich, R. (2013): Zukunftsforschung für Gesellschaft und Wirtschaft. In: Zweck, A. \& Popp, R. (Hrsg.): Zukunftsforschung im Praxistest, 353-384, Wiesbaden: Springer.

Milbert, A. (2013): Vom Konzept der Nachhaltigkeitsindikatoren zum System der regionalen Nachhaltigkeit. In: Bundesinstitut für Bau-, Stadt- und Raumforschung (BBSR) im Bundesamt für Bauwesen und Raumordnung (BBR) (Hrsg.): Informationen zur Raumentwicklung, 2013 (1), 37-50.

Niggli, U.; Riedel, J.; Brühl, C.; Liess, M.; Schulz, R.; Altenburger, R.; Märländer, B.; Bokelmann, W.; Heß, J.; Reineke, A.; Gerowitt, B. (2020): Pflanzenschutz und Biodiversität in Agrarökosystemen. In: Berichte über Landwirtschaft - Zeitschrift für Agrarpolitik und Landwirtschaft, 98 (1), 1-39. Verfügbar unter https://buel.bmel.de/index.php/buel/article/view/272/481, abgerufen am 12.03.2021.

Peter, C. \& Swilling, M. (2014): Linking Complexity and Sustainability Theories: Implications for Modeling Sustainability Transitions. In: Sustainability, 6 (3), 1594-1622.

Pillkahn, U. (2013): Pictures of the Future. Zukunftsbetrachtungen im Unternehmensumfeld. In: Zweck, A. \& Popp, R. (Hrsg.), Zukunftsforschung im Praxistest, 41-80, Wiesbaden: Springer. 
Ratter, B. M. W. \& Treiling, T. (2008): Komplexität - oder was bedeuten die Pfeile zwischen den Kästchen? In: Egner, H.; Ratter, B. M. W. \& Dikau, R. (Hrsg.): Umwelt als System - System als Umwelt? Systemtheorien auf dem Prüfstand, 23-38, München: Oekom.

Sandercock, L. (2010): From the Campfire to the Computer: An Epistemology of Multiplicity and the Story Turn in Planning. In: Sandercock, L. \& Attili, G. (Hrsg.): Multimedia explorations in urban policy and planning. Beyond the flatlands, 17-38, Dordrecht: Springer Netherland.

Skrimizea, E., Haniotou, H. \& Parra, C. (2019): On the 'complexity turn' in planning: An adaptive rationale to navigate spaces and times of uncertainty. In: Planning Theory, 18 (1), 122-142.

Steele, L. (1991): Towards a Theory of Emergent Functionality. In: Meyer, J.-A. \& Wilson, S. W. (Hrsg.): From animals to animats. Proceedings of the First International Conference on Simulation of Adaptive Behavior, 451-461, Cambridge, Mass.: MIT Press.

Tippett, J. (2010): Going Beyond the Metaphor of the Machine: Complexity and Participatory Ecological Design. In Roo, G. de \& Silva, E. A. (Hrsg.): A Planner's Encounter with Complexity. New Directions in Planning Theory, 237-262, Florence: Taylor and Francis.

Vester, F. (2002): Die Kunst vernetzt zu denken: Ideen und Werkzeuge für einen neuen Umgang mit Komplexität, Ein Bericht an den Club of Rome. München: Deutscher Taschenbuchverlag.

Weig, B. (2016): Resilienz komplexer Regionalsysteme. Brunsbüttel zwischen Lock-in und Lernprozessen. Wiesbaden: Springer Spektrum.

Wolf, I. (2020): Soziales Nachhaltigkeitsbarometer der Energiewende 2019. Kernaussagen und Zusammenfassung der wesentlichen Ergebnisse. https://www.iass-potsdam.de/sites/default/files/2020-04/0nline_IASS_Barometer_200422_FINALFINAL.pdf, abgerufen am 08.03.2021.

\section{Kontakt}

Prof. Dr.-Ing. Gerhard Glatzel

g.glatzel@hbk-bs.de

Dr. Mehdi Mozuni

m.mozuni@hbk-bs.de

Maren Ohlhoff

m.ohlhoff@hbk-bs.de

Institut für Designforschung

Hochschule für Bildende Künste Braunschweig

Johannes-Selenka-Platz 1

38118 Braunschweig 